\title{
Qubit decoherence due to detector switching
}

\author{
Ioana Serban ${ }^{1,2}$ and Frank K Wilhelm ${ }^{2,3 *}$
}

${ }^{*}$ Correspondence:

fwm@lusi.uni-sb.de

${ }^{2}$ IQC and Department of Physics

and Astronomy, University of

Waterloo, Waterloo, Ontario, Canada

${ }^{3}$ Theoretical Physics, Saarland

University, Saarbrücken, Germany

Full list of author information is

available at the end of the article

\begin{abstract}
We discuss the noise occurring during a classical dissipative switching process as it would be detected by a quantum bit used as a detector for noise. In particular we study the switching-induced decoherence during escape events. We present a simple method to obtain analytical results for the qubit dephasing and bit-flip, which goes beyond the simple Born-Markov notion of the qubit as a spectrometer for noise but still allows us to correlate its behavior with the noise source. These results also provide insight into the qubit measurement process involving a switching type of detector, showing under which conditions switching detectors can be operated fast and with low error. In particular, the state in the end recovers from temporary bit flip errors due to an intrinsic approximate time reversal symmetry, whereas the switching process mostly produces low frequency pure dephasing noise.
\end{abstract}

Keywords: switching process; noise; qubit

\section{Introduction}

Noise-activated switching out of a metastable state is a common phenomenon in a wide range of physical systems, including Josephson junctions, nanomechanical devices, and chemical reactions [1,2]. Starting with Kramers seminal work [3], such processes have been studied close to equilibrium [4], as well as in driven systems [5]. The activated escape paths have been studied theoretically and observed experimentally [6,7]. Due to the multiple time scales involved, some of them very fast, observing these fluctuations remains challenging.

Recently, noise-activated switching has gained attention due to its role in qubit detection. Examples of switching detectors include the superconducting quantum interference device (SQUID) [8-10], where switching occurs between the superconducting and dissipative state and is driven by quantum fluctuations, and the Josephson bifurcation amplifier (JBA) [11-14], which has been employed in the delicate task of detecting a qubit state in a minimally invasive fashion [15]. In this case, the detector can switch between different, weakly dissipative, dynamical states based on quantum activation [16] or dynamical tunneling [17]. Using an appropriate choice of a reference frame, switching between such dynamical states can also be described as escape from a static metastable well [16, 17]. Switching detectors are currently receiving renewed attention for their potential as microwave photon counters [18-20].

Switching is a highly nonlinear phenomenon, driven by large, non-equilibrium environmental fluctuations. Consequently, the noise produced during a switching process has long correlation times and can be very large. Its impact on a qubit is thus far outside the

(c) 2015 Serban and Wilhelm; licensee Springer. This is an Open Access article distributed under the terms of the Creative Commons Attribution License (http://creativecommons.org/licenses/by/4.0), which permits unrestricted use, distribution, and reproduction in any medium, provided the original work is properly credited. 
Markov paradigm and detection is far from the weak measurement scenario. Some understanding for switching-type detectors has been provided by numerical studies [21, 22] and in a simplified two-state detector version in Refs. [23, 24]. General properties of binary outcome detectors have been discussed in [25]. Our approach is complementary in taking a rather concrete model of the detector.

In this paper we propose a simple analytical method to investigate qubit decoherence due to a switching process that can be part of a detector. Qubits are excellent spectrometers for quantum noise, most simply based on studying their relaxation rate [26], with a bandwidth that can be extended when dephasing and echo data are also taken into account [27]. Here, we model the noise source as a classical, overdamped particle trapped in a metastable well. This model is a strong simplification of the various detectors described above, yet capturing essential elements of the underlying switching process. These elements include that switching is a rare event and is driven by fluctuations, either thermal or quantum. Here, the escape of the particle is driven by large, rare fluctuations in the environment. We investigate the qubit dephasing and bit flips induced by the switching during the escape event. We adopt the simple model of quantum measurement that dephasing is a necessary ingredient as the real-time representation of wavefunction collapse, whereas bit-flips are unwanted falsifications of the measurement outcome. Note that these bit-flips occur if the qubit Hamiltonian and its coupling to the detector do not commute. They can be seen as an interplay of the coherent evolution of the qubit during the measurement process and the noise that is inevitable during detection. Note that an actual detector does not have to switch - in the 'down' state, the detector potential is deformed to make switching highly unlikely, leading to another class of trajectories that stay confined during the time of the experiment. Note that the trajectories studies here are entirely classical, quantum physics only comes in by the qubit exposed to them, making our approach different from the quantum trajectories method [28].

\section{Method}

The overdamped classical particle performs Brownian motion according to

$$
\dot{x}=K(x)+f(t),
$$

where $K$ is the deterministic force experienced by the particle resulting from the metastable potential and $f$ is white Gaussian noise with a probability density functional given by [29]

$$
P[f(t)]=\exp \left(-\int_{0}^{t_{\mathrm{f}}} \frac{f^{2}(t)}{2 D} d t\right)
$$

where we assume [30] the noise intensity $D$ to be small compared to the barrier height $\Delta U$, see Figure 1 . The probability density functional is not normalized a priori, subsequent expressions have to be normalized by hand. For a noise driven trajectory it can be obtained by substituting Eq. (1)

$$
P[x(t)]=\exp \left(-\frac{S[x(t)]}{D}\right), \quad S[x(t)]=\frac{1}{2} \int_{0}^{t_{f}} d t(\dot{x}-K(x))^{2} .
$$




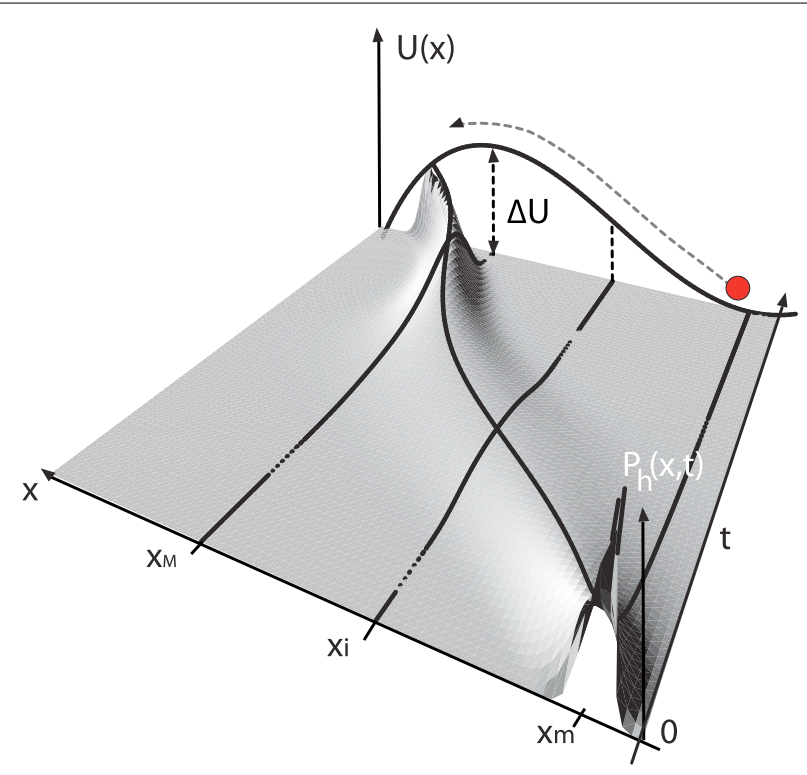

Figure 1 Prehistory probability density $P(x, t)$ for the potential $U(x)$, where $U^{\prime}(x)=-K(x)$, and optimal trajectory $\boldsymbol{x}_{\mathbf{o p t}}(\boldsymbol{t})$. Here $x_{\mathrm{m}, \mathrm{M}, \mathrm{i}}$ are the positions of the minimum, maximum and inflection points of the potential.

For the study of qubit decoherence during a switching event one will need expectation values for a generic observable $O$ of the type

$$
\begin{aligned}
& O\left(t_{0}\right)=\left\langle\exp \left(\lambda \phi\left[x(t), s\left(t, t_{0}\right)\right]\right)\right\rangle_{\mathrm{sw}}, \\
& \phi\left[x(t), s\left(t, t_{0}\right)\right]=\int_{0}^{t_{\mathrm{f}}} x(t) s\left(t, t_{0}\right) d t
\end{aligned}
$$

were $s\left(t, t_{0}\right)$ is a time dependent generating field for computing dephasing and bit-flip as will be detailed later. The coupling constant $\lambda$ has been singled out to parameterize the coupling to the qubit and the time $t_{0}$ indicates when the observable is measured. Effectively, it constitutes a modulation of $x(t)$ and $\lambda$. This approach is fairly standard in stochastic path integrals and the choice of $s\left(t, t_{0}\right)$ suitable for our problem will be clarified later. This formulation allows for the present formalism to include detectors such as the JBA, where, in a rotating frame, the coupling between the qubit and detector becomes time dependent and periodic. We are interested in the qubit decoherence during switching. Thus, the averaging \langle\rangle$_{\mathrm{sw}}$ is performed only over switching trajectories of the detector, which satisfy the boundary conditions $x(0)=x_{\mathrm{m}}$ and $x\left(t_{\mathrm{f}}\right)=x_{\mathrm{f}}$, with $x_{\mathrm{m}}$ inside and $x_{\mathrm{f}}$ outside the metastable well. By choosing $s\left(t, t_{0}\right)=0$ at $t_{\mathrm{f}}>t>t_{0}$, the average becomes postconditioned by a switching event having taken place at the final time $t_{\mathrm{f}}$, i.e., experimental verification would need to include recording the time of the switch and post-selecting for different times. This condition makes sure that we only study realizations of the measurement when switching occurs. In order to not over-constrain the trajectories we have verified the dependence of our result on the precise choice of $x_{\mathrm{f}}$ and found minuscule changes only. 
Since the exact switching trajectory between the initial and final point remain random, we average over all possible paths using the weight $e^{-S[x] / D}$

$$
\begin{aligned}
O\left(t_{0}\right)= & \int_{\left(x_{\mathrm{m}}, 0\right)}^{\left(x_{\mathrm{f}}, t_{\mathrm{f}}\right)} \mathcal{D} x(t) \exp \left(\lambda \phi\left[x(t), s\left(t, t_{0}\right)\right]-\frac{S[x(t)]}{D}\right) \\
& \times P\left(x_{\mathrm{m}}, 0 \mid x_{\mathrm{f}}, t_{\mathrm{f}}\right)^{-1}
\end{aligned}
$$

where the total switching probability

$$
P\left(x_{\mathrm{m}}, 0 \mid x_{\mathrm{f}}, t_{\mathrm{f}}\right)=\int_{\left(x_{\mathrm{m}}, 0\right)}^{\left(x_{\mathrm{f}}, \mathrm{t}_{\mathrm{f}}\right)} \mathcal{D} x(t) \exp \left(-\frac{S[x(t)]}{D}\right)
$$

serves as a normalization.

Switching over a high barrier is a rare event and thus requires very specific noise realizations. Thus, the switching trajectories form a narrow tube in phase space centered around an optimal trajectory $[31,32]$ which minimizes $S$, and for the present case satisfies

$$
\ddot{x}_{\mathrm{opt}}=K\left(x_{\mathrm{opt}}\right) K^{\prime}\left(x_{\mathrm{opt}}\right), \quad x_{\mathrm{opt}}(0)=x_{\mathrm{m}}, \quad x_{\mathrm{opt}}\left(t_{\mathrm{f}}\right)=x_{\mathrm{f}} .
$$

This optimal trajectory is driven by an optimal realization of the environmental noise. Thus $S[x(t)]=S\left[x_{\mathrm{opt}}(t)\right]+S_{2}\left[x(t)-x_{\mathrm{opt}}(t)\right]$ and we perform a saddlepoint approximation around this optimal solution

$$
S_{2}[x(t)] \approx \frac{1}{2} \int_{0}^{t_{\mathrm{f}}} d t\left(\dot{x}(t)^{2}-\Lambda(t)^{2} x(t)^{2}\right)
$$

where $\Lambda(t)^{2}=-\left.\left(K^{\prime}(x)^{2}+K(x) K^{\prime \prime}(x)\right)\right|_{x=x_{\text {opt }}(t)}$. Divergences due to the emergence of a slow mode on the barrier top [30,33] are avoided by the appropriate choice of the initial (kinetic) energy, $0<\dot{x}(0)^{2} / 2 \ll \Delta U$ in order to satisfy the boundary conditions (8). Thus, the switching event takes place, with non-vanishing probability, within a finite time $t_{\mathrm{f}}$. Within the saddle point approximation (9), the path integral (6) becomes Gaussian and can be evaluated analytically [34]

$$
O\left(t_{0}\right)=\exp \left(\lambda \phi\left[x_{\mathrm{opt}}(t)+\frac{x_{0}(t)}{2}, s\left(t, t_{0}\right)\right]\right)
$$

where $x_{0}$ is the solution of

$$
\ddot{x}_{0}+\Lambda^{2} x_{0}+D \lambda s\left(t, t_{0}\right)=0, \quad x_{0}(0)=x_{0}\left(t_{\mathrm{f}}\right)=0 .
$$

The two linearly independent solutions of the homogeneous part of Eq. (11) are

$$
x_{1}(t)=\dot{x}_{\mathrm{opt}}(t), \quad x_{2}(t)=\dot{x}_{\mathrm{opt}}(t) \int_{0}^{t} \frac{d t^{\prime}}{x_{\mathrm{opt}}^{2}\left(t^{\prime}\right)}
$$

and the full $x_{0}(t)=x_{1}(t) c_{1}(t)+x_{2}(t) c_{2}(t)$ can be determined by variation of parameters.

We consider the case of a potential given by $U(x) / \Omega=x / 2-x^{3} / 6$, which can, for example, approximate a Josephson junction biased at half the critical current. Note that this 
approximation does not account for retrapping of the Josephson junction in the supercurrent state after $n$ full rotations of the phase, hence it applies when the bias is large enough to precvent such retracing or phase diffusion. $\Omega=K^{\prime}\left(x_{\mathrm{m}}\right)$ plays the role of a characteristic frequency in our effective system but physically corresponds to the damping coefficient.

\section{Results}

Pure dephasing: We are now going to discuss the impact of these distributions on dephasing and bit-flip. The stochastic process and distribution of trajectories is the same in both cases. Dephasing typically probes the low-frequency part of the noise spectrum. We assume a qubit coupling Hamiltonian of the form

$$
\hat{H}=\hbar \omega \hat{\sigma}_{z}+\eta_{d} \hat{\sigma}_{z} x(t)
$$

where $x(t)$ is the coordinate of the classical particle. The only effect of the environment on the qubit in this case is the irreversible decay of the phase coherence $C\left(t_{0}\right)=O\left(t_{0}\right)$, which can be obtained by replacing in Eq. (4) $\lambda=\lambda_{d}=\eta_{d} /(i \hbar)$ and the generating function

$$
s\left(t, t_{0}\right)= \begin{cases}1, & t<t_{0}, \\ 0, & t>t_{0}\end{cases}
$$

which has been chosen to fix the initial condition of the particle. This choice can be physically understood to collect all low-frequency fluctuations in the past of the evaluation of the observable at time $t$.

Figure 2 shows that the escape process is driven by strong noise (c), with a maximum intensity at the time where the most probable trajectory (d) reaches the inflection point of the barrier. In the vicinity of the same point, a sharp drop in qubit coherence (a) is observed. After the inflection point the motion is slowed down, and becomes diffusive close to the barrier top. During this stage, the qubit coherence remains at an almost constant value. We observe that the optimal noise becomes stronger for shorter values of $t_{\mathrm{f}}$. However, the strongest drop in qubit coherence was observed for the longest $t_{\mathrm{f}}$. In this case the
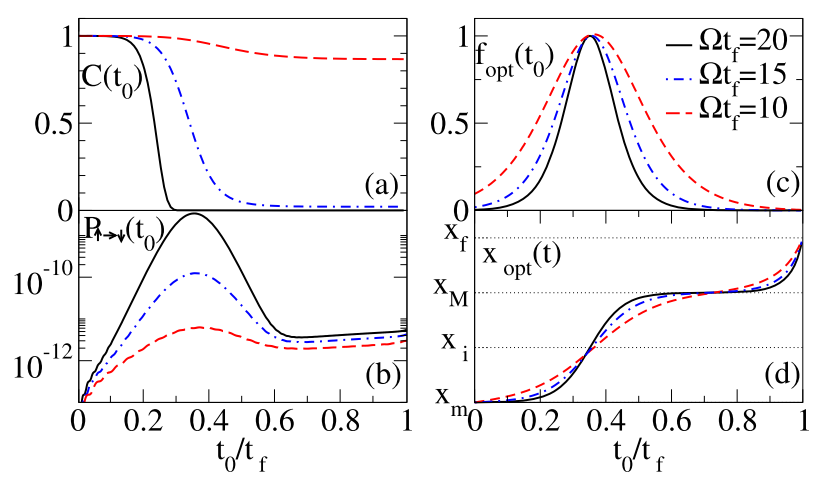

Figure 2 Qubit coherence during a switching event $\left(t_{0}<t_{f}\right)(a)$, probability for an induced bit flip error as a function of $t_{0}(b)$, the corresponding most probable noise trajectory $f_{\text {opt }}\left(t_{0}\right)$ and (c) most probable switching trajectory $x_{\mathbf{o p t}}\left(t_{0}\right)(\mathbf{d})$ for various values of $\boldsymbol{t}_{\mathbf{f}}$. The particle starts from the minimum of the well with a speed adjusted to the switching condition and a diffusion coefficient $D=0.01$. 
optimal trajectory spends more time close to the barrier top, where the motion is diffusive and broadly distributed, driven by low amplitude noise.

Bit flips: We consider a qubit-environment coupling which allows for bit flips

$$
\hat{H}=\hbar \omega \hat{\sigma}_{z}+\eta_{f} x(t) \hat{\sigma}_{x}
$$

Bit-flip typically probes the noise spectrum around the qubit frequency. Under this generic scenario, the interplay of unitary qubit evolution and the measurement not being instantaneous leads to bit-flips compromising the post-measurement state. We concentrate on the case of that unwanted evolution being slow, i.e., weak qubit-detector coupling. The probability of noise induced transitions during a switching event at $t_{0}<t_{\mathrm{f}}$ is given by the propagator in the interaction picture (henceforth labelled by a subscript $I$ )

$$
P_{\uparrow \rightarrow \downarrow}\left(t_{0}\right)=\left|\left\langle\downarrow\left|\hat{U}_{I}\left(t_{0}\right)\right| \uparrow\right\rangle\right|^{2} P\left(x_{\mathrm{m}}, 0 \mid x_{\mathrm{f}}, t_{\mathrm{f}}\right)^{-1},
$$

where in the limit of short time and weak coupling

$$
\begin{aligned}
& \hat{U}_{I}\left(t_{0}\right)=\mathcal{T} \exp \left(\int_{0}^{t_{0}} d t \frac{H_{I}(t)}{i \hbar}\right) \approx 1+\int_{0}^{t_{0}} d t \frac{H_{I}(t)}{i \hbar}, \\
& \hat{H}_{I}(t)=\eta_{f} \hat{U}_{0}^{\dagger}(t) \hat{\sigma}_{x} \hat{U}_{0}(t) x(t)
\end{aligned}
$$

and $\hat{U}_{0}$ describes the free qubit evolution. We follow the standard steps of relaxation calculations by time-dependent perturbation theory as outlines in $[26,35]$ but do not perform the Markov approximation which would be inappropriate given the strong temporal correlations of the noise. Still, the transition probability can be expressed through the autocorrelation function of $\phi$ which in turn can be generated out of $O$ by formal differentiation of Eq. (4). We obtain

$$
\begin{aligned}
P_{\uparrow \rightarrow \downarrow}\left(t_{0}\right)= & \lim _{\lambda_{f} \rightarrow 0} \frac{\partial^{2}}{\partial \lambda_{f}^{2}} \frac{\eta_{f}^{2}}{\hbar^{2}}\left\langle\exp \left(\lambda_{f} \phi\left[x(t), s\left(t, t_{0}\right) \mathcal{R}(t)\right]\right)\right. \\
& \left.+\exp \left(\lambda_{f} \phi\left[x(t), s\left(t, t_{0}\right) \mathcal{I}(t)\right]\right)\right\rangle_{\mathrm{sw}}, \\
\mathcal{R}(t)+i \mathcal{I}(t)= & \left\langle\downarrow\left|\hat{U}_{0}^{\dagger}(t) \hat{\sigma}_{x} \hat{U}_{0}(t)\right| \uparrow\right\rangle .
\end{aligned}
$$

In Figure 2(b) we observe, again, a sharp feature in $P_{\uparrow \rightarrow \downarrow}\left(t_{0}\right)$ at the point in time where the most probable trajectory $(\mathrm{d})$ reaches the steepest point on the potential barrier, and the driving noise (c) reaches it maximum strength. Despite the optimal noise being strongest for short switching time $t_{\mathrm{f}}$, the peak in $P_{\uparrow \rightarrow \downarrow}\left(t_{0}\right)$ is stronger for longer $t_{\mathrm{f}}$. Another notable feature is the quasi-reversion of the bit flip error which occurs at this point. This feature cannot be explained by the single, deterministic trajectory $x_{\mathrm{opt}}$ alone, which causes only the steady increase of $P_{\uparrow \rightarrow \downarrow}\left(t_{0}\right)$.

\section{Discussion}

The results presented above can be understood from the distribution of switching trajectories. We calculate the probability $P_{h}(x, t)$ for the classical particle to occupy the position 
$x$ at time $t$ during a switching event, i.e., a prehistory density distribution [30]

$$
P_{h}(x, t)=\frac{P\left(x_{\mathrm{m}}, 0 \mid x, t\right) P\left(x, t \mid x_{\mathrm{f}}, t_{\mathrm{f}}\right)}{P\left(x_{\mathrm{m}}, 0 \mid x_{\mathrm{f}}, t_{\mathrm{f}}\right)} .
$$

Within the approximation (9), the probability for a transition between any pair of points $\left(x_{1}, t_{1}\right)$ and $\left(x_{2}, t_{2}\right)$, with $t_{1,2}<t_{\mathrm{f}}$ reads

$$
P\left(x_{1}, t_{1} \mid x_{2}, t_{2}\right)=\int_{\left(\delta x_{1}, t_{1}\right)}^{\left(\delta x_{2}, t_{2}\right)} \mathcal{D} x(t) \exp \left(-\frac{S\left[x_{\mathrm{opt}}(t)\right]_{1}^{2}+S_{2}[x(t)]_{1}^{2}}{D}\right)
$$

where $S[x(t)]_{1}^{2}$ implies that the time integral is taken between $t_{1}$ and $t_{2}$ and $\delta x_{1,2}=x_{1,2}-$ $x_{\mathrm{opt}}\left(t_{1,2}\right)$. One can show that

$$
P\left(x_{1}, t_{1} \mid x_{2}, t_{2}\right)=\exp \left(-\frac{S\left[x_{\mathrm{opt}}(t)\right]_{1}^{2}+S_{2}\left[x_{b}(t)\right]_{1}^{2}}{D}\right) F\left(t_{1} \mid t_{2}\right),
$$

where $\ddot{x}_{b}+\Lambda^{2}(t) x_{b}(t)=0$ and $x_{b}\left(t_{1,2}\right)=\delta x_{1,2}$ and

$$
\begin{aligned}
F\left(t_{1} \mid t_{2}\right) & =\int_{\left(0, t_{1}\right)}^{\left(0, t_{2}\right)} \mathcal{D} x(t) \exp \left(-\frac{S_{2}[x(t)]}{D}\right) \\
& =\left(2 \pi D \dot{x}_{\mathrm{opt}}\left(t_{1}\right) \dot{x}_{\mathrm{opt}}\left(t_{2}\right) \int_{t_{1}}^{t_{2}} \frac{d t}{\dot{x}_{\mathrm{opt}}^{2}(t)}\right)^{-1 / 2} .
\end{aligned}
$$

We obtain a Gaussian distribution, centered around $x_{\mathrm{opt}}(t)$

$$
P_{h}(x, t)=\frac{1}{\sqrt{\pi w(t)}} \exp \left(-\frac{\left(x-x_{\mathrm{opt}}(t)\right)^{2}}{w(t)}\right)
$$

where $w(t)=F\left(0 \mid t_{\mathrm{f}}\right)^{2} /\left(F(0 \mid t)^{2} F\left(t \mid t_{\mathrm{f}}\right)^{2}\right)$.

Figures 1 and 3 reveal a narrow tube of trajectories close to the bottom of the well, followed by a strong widening of the distribution in the process of climbing up the potential

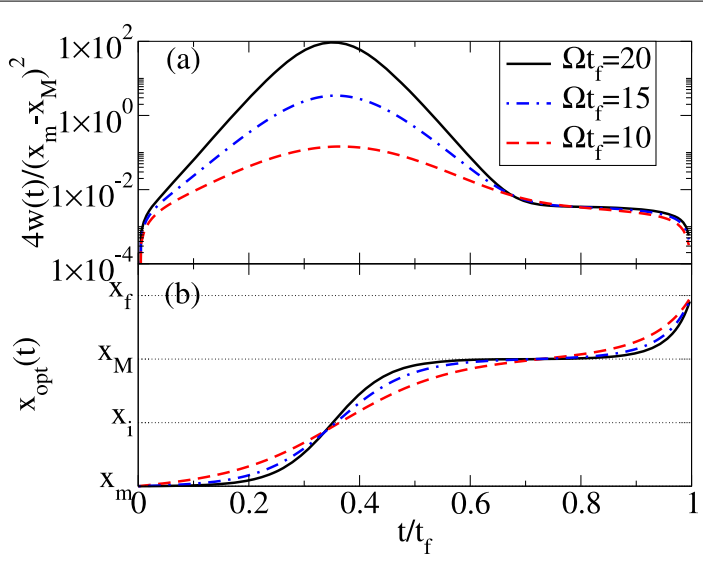

Figure 3 Width of the prehistory probability distribution $P_{h}(x, t)(a)$ and optimal trajectory (b) for a particle starting from the minimum at rest, parameters as in previous figure. 
barrier. This event is driven by a sharp noise pulse. On the barrier top we see again a fairly localized density distribution, again driven by low-amplitude noise. The tube narrows even more on the outer side of the barrier. These results are in agreement with the findings of Ref. [6], for a different system. It should be emphasized that this narrow tube is due to the fact that the necessary realization of environmental noise is a rare event in sync with the connection to a fixed final time - if the latter were not imposed, we would find a mixture of those tubes.

We found, see Figure 2 that the qubit suffers the strongest decoherence at the point in time when the optimal trajectory reaches the steepest point on the barrier wall. This is true for both bit flips and dephasing. The magnitude of both effects depends strongly on the total time necessary for the switching event, such that longer $t_{\mathrm{f}}$ leads to enhanced coherence loss, and higher bit flip rate. The observed effect can be explained by the strong widening of the prehistory distribution $P_{h}(x, t)$ at the same point in time, see Figure 3.

The peak in $P_{\uparrow \rightarrow \downarrow}$ originates in the strong widening of the trajectory tube. Thus, large excursions around $x_{\mathrm{opt}}$, see Figure 1 , are very probable during this time. However, since each of these switching trajectories must return to the narrow tube of trajectories on the other side of the widening, i.e. in the region close to the barrier top, they all show an approximate time reversal symmetry. In other words, part of the change to the qubit state - the one originating from the average of the trajectory, is deterministic and is in parts undoing itself. Thus any induced bit flip errors will be partially reversed when the particle reaches the barrier top.

In conclusion, we have studied the interaction between a switching particle that could be part of a detector with a qubit. We have shown how the stochastic dynamics of the detector are correlated with the qubit. A switching detector, such as the one modeled here, presents several qualities which makes it desirable as a nonlinear qubit detector. The strong decoherence suffered by the qubit as the classical particle climbs up the potential barrier affects strongly the coherence, leading to a fast measurement. The equally strong bit flip errors acquired during the process are reversed by the quasi time-reversal symmetry of most trajectories.

We observe that a fast switching event causes less decoherence, despite stronger noise being required for surmounting the barrier. Figure 3 reveals that longer switching time $t_{\mathrm{f}}$ allows more freedom in the choice of the particular time the particle climbs up the potential barrier and such incoherent behavior causes decoherence between different realizations of the experiment.

We note that the widening of the trajectory tube at the inflection point of the potential appears throughout the literature on Brownian motion as a general feature of the tube of escape trajectories out of metastable states, but the connection to quantum measurement has not been made yet. Having identified it as the major cause for the features observed in qubit decoherence, we expect these features to be common to various potentials. Therefore we expect that our results have applicability to existing experimental setups, e.g. the JBA and the DC-SQUID. 


\section{Author details}

${ }^{1}$ Instituut Lorentz, Leiden University, Leiden, The Netherlands. ${ }^{2}$ IQC and Department of Physics and Astronomy, University of Waterloo, Waterloo, Ontario, Canada. ${ }^{3}$ Theoretical Physics, Saarland University, Saarbrücken, Germany.

\section{Acknowledgements}

We are grateful to MI Dykman for pointing out the prehistory probability distribution approach and for many useful suggestions. We acknowledge useful discussions with J Gambetta, WA Coish and TC Wei as well as LCG Govia for his careful reading of the manuscript. This work has been supported by NSERC Discovery Grants and Quantum Works.

Supported by the Army Research Office under contract W911NF-14-1-0080.

\section{Received: 23 October 2014 Accepted: 22 January 2015 Published online: 07 March 2015}

\section{References}

1. Aldridge JS, Cleland AN. Noise-enabled precision measurements of a Duffing nanomechanical resonator. Phys Rev Lett. 2005;94:156403.

2. Wales D. Energy landscapes: applications to clusters, biomolecules and glasses. Cambridge: Cambridge University Press; 2003.

3. Kramers H. Brownian motion in a field of force and the diffusion model of chemical reactions. Physica. 1940;7:284.

4. Hanggi P, Talkner P, Borkovec M. Reaction rate theory: fifty years after Kramers. Rev Mod Phys. 1990;62:252.

5. Lehmann J, Reimann P, Hänggi P. Surmounting oscillating barriers. Phys Rev Lett. 2000;84:1639.

6. Hales J, Zhukov A, Roy R, Dykman MI. Dynamics of activated escape, and its observation in a semiconductor laser. Phys Rev Lett. 2000;85:78.

7. Chan HB, Dykman MI, Stambaugh C. Paths of fluctuation induced switching. Phys Rev Lett. 2008;100:130602.

8. van der Wal C, ter Haar A, Wilhelm F, Schouten R, Harmans C, Orlando T, Lloyd S, Mooij J. Quantum superposition of macroscopic persistent-current states. Science. 2000;290:773.

9. Vion D, Aassime A, Cottet A, Joyez P, Pothier H, Urbina C, Esteve D, Devoret M. Manipulating the quantum state of an electrical circuit. Science. 2002;296:866

10. Martinis JM, Nam S, Aumentado J, Urbina C. Rabi oscillations in a large Josephson-junction qubit. Phys Rev Lett. 2002;89:117901.

11. Siddiqi I, Vijay R, Pierre F, Wilson CM, Metcalfe M, Rigetti C, Frunzio L, Devoret MH. RF-driven Josephson bifurcation amplifier for quantum measurement. Phys Rev Lett. 2004;93:207002.

12. Siddiqi I, Vijay R, Pierre F, Wilson CM, Frunzio L, Metcalfe M, Rigetti C, Schoelkopf RJ, Devoret MH, Vion D, Esteve D. Direct observation of dynamical bifurcation between two driven oscillation states of a Josephson junction. Phys Rev Lett. 2005;94:027005.

13. Metcalfe M, Boaknin E, Manucharyan V, Vijay R, Siddiqi I, Rigetti C, Frunzio L, Schoelkopf RJ, Devoret MH. Measuring the decoherence of a quantronium qubit with the cavity bifurcation amplifier. Phys Rev B. 2007;76:174516.

14. Schmitt V, Zhou X, Juliusson K, Blais A, Bertet P, Vion D, Esteve D. Multiplexed Readout of transmon qubits with Josephson bifurcation amplifiers. Phys Rev A. 2008;90:062333.

15. Lupascu A, Saito S, Picot T, de Groot PC, Harmans CJPM, Mooij JE. Quantum non-demolition measurement of a superconducting two-level system. Nat Phys. 2007;3:119.

16. Marthaler M, Dykman M. Switching via quantum activation: a parametrically modulated oscillator. Phys Rev A. 2006;73:042108

17. Serban I, Wilhelm FK. Dynamical tunneling in macroscopic systems. Phys Rev Lett. 2007;99:137001.

18. Chen Y-F, Hover D, Sendelbach S, Maurer L, Merkel ST, Pritchett EJ, Wilhelm FK, McDermott R. Microwave photon counter based on Josephson junctions. Phys Rev Lett. 2011;107:217401.

19. Poudel A, McDermott R, Vavilov MG. Quantum efficiency of a microwave photon detector based on a current-biased Josephson junction. Phys Rev B. 2012;86:174506.

20. Goiva LCG, Pritchett EJ, Xu C, Plourde BLT, Vavilov MG, Wilhelm FK, McDermott R. High-fidelity qubit measurement with a microwave-photon counter. Phys Rev A. 2014;90:062307.

21. Nakano H, Saito S, Semba K, Takayanagi H. Quantum time-evolution in qubit readout process with a Josephson bifurcation amplifier. Phys Rev Lett. 2009;102:257003.

22. Nakano H, Tanaka H, Saito S, Semba K, Takayanagi H, Ueda M. A theoretical analysis of flux-qubit measurements with a dc-SQUID (unpublished). arXiv:cond-mat/0406622.

23. Ashhab S, You JQ, Nori F. Weak and strong measurement of a qubit using a switching-based detector. Phys Rev $A$. 2009;79:032317

24. Ashhab S, You JQ, Nori F. The information about the state of a qubit gained by a weakly coupled detector. New J Phys. 2009;11:083017.

25. Korotkov AN. Weak and strong measurement of a qubit using a switching-based detector. Phys Rev B. 2008;78:174512.

26. Schoelkopf RJ, Clerk AA, Girvin SM, Lehnert KW, Devoret MH. Qubits as spectrometers of quantum noise. In: Nazarov YV, Blanter YM, editors. Quantum noise. Dordrecht: Kluwer Academic; 2006.

27. Bylander J, Gustavsson S, Yan F, Yoshihara F, Harrabi K, Fitch G, Cory DG, Nakamura Y, Tsai J-S, Oliver WD. Noise spectroscopy through dynamical decoupling with a superconducting flux qubit. Nat Phys. 2011;7:565.

28. Wiseman HM, Milburn GJ. Quantum measurement and control. Cambridge: Cambridge University Press; 2014.

29. Feynman R, Hibbs A. Quantum mechanics and path integrals. New York: McGraw-Hill; 1965.

30. Dykman MI, McClintock PVE, Smelyanski VN, Stein ND, Stocks NG. Optimal paths and the pre-history problem for large fluctuations in noise-driven systems. Phys Rev Lett. 1992;68:2718.

31. Onsager L, Machlup S. Fluctuations and irreversible processes. Phys Rev. 1953;91:1505.

32. Dykman M, Krivoglaz M, Soskin S. Transition probabilities and spectral density of fluctuations of noise-driven bistable systems. In: Moss F, McClintock PVE, editors. Noise in nonlinear dynamical systems. Cambridge: Cambridge University Press; 1989. p. 347.

33. Luchinsky D, McClintock P, Dykman M. Analogue studies of nonlinear systems. Rep Prog Phys. 1998;61:889. 
34. Kleinert H. Path integrals in quantum mechanics, statistics, polymer physics, and financial markets. Singapore: World Scientific; 2006.

35. Wilhelm FK, Hartmann U, Storcz MJ, Geller MR. Quantum computing with superconductors II: decoherence in manipulating quantum coherence. In: Flatte ME, Tifrea I, editors. Solid state systems. Dordrecht: Springer; 2006. p. 195.

Submit your manuscript to a SpringerOpen ${ }^{\circ}$ journal and benefit from:

- Convenient online submission

Rigorous peer review

- Immediate publication on acceptance

- Open access: articles freely available online

- High visibility within the field

- Retaining the copyright to your article

Submit your next manuscript at $\boldsymbol{s p r i n g e r o p e n . c o m ~}$ 